\title{
Prix Fermat 1989
}

Annales de la faculté des sciences de Toulouse $5^{e}$ série, tome 11, $\mathrm{n}^{\circ} 2$ (1990), p. 7-8

$<$ http://www.numdam.org/item?id=AFST_1990_5_11_2_7_0>

(C) Université Paul Sabatier, 1990, tous droits réservés.

L'accès aux archives de la revue «Annales de la faculté des sciences de Toulouse » (http://picard.ups-tlse.fr/ annales/) implique l'accord avec les conditions générales d'utilisation (http://www.numdam.org/conditions). Toute utilisation commerciale ou impression systématique est constitutive d'une infraction pénale. Toute copie ou impression de ce fichier doit contenir la présente mention de copyright.

\section{Numdam}

Article numérisé dans le cadre du programme

Numérisation de documents anciens mathématiques

http://www.numdam.org/ 


\section{Prix Fermat 1989}

Organisé par l'Université Paul Sabatier de Toulouse et Matra Espace, le prix Fermat de recherches en mathématiques 1989 a été décerné conjointement à :

\section{Monsieur A. BAHRI pour l'introduction de méthodes nouvelles en calcul des variations; \\ Monsieur K.A. RIBET pour sa contribution à la théorie des nombres et au problème de Fermat.}

Un extrait de la citation du Jury ainsi qu'un article de K.A. BAHRI (collaboration avec P. Rabinowitz) paraissent dans ce numéro des Annales de la Faculté des Sciences de Toulouse. Il en a été de même pour K.A. RIBET dans un précédent numéro.

"Né en Tunisie en 1955, Abbas Bahri a reçu son éducation supérieure en France : École Normale Supérieure de la rue d'Ulm, Agrégation de Mathématiques et Doctorat d'État (soutenu en 1981). Après un séjour à l'Université de Chicago, il est nommé en 1984 Maître de Conférence à l'École Polytechnique. En 1987 il accepte une offre de "Full Professor" à l'Université de Rutgers aux USA. Il est bien connu, surtout depuis les travaux de P. Fermat, que le Calcul des Variations (principe de moindre action) joue un rôle essentiel dans les domaines des Mathématiques liés à la Physique et à la Mécanique.

A. BAHRI s'est attaqué à des problèmes variationnels réputés difficiles qu'il a abordé avec courage et imagination. Il a réalisé des progrès très importants grâce à un outil puissant qu'il a développé : la méthode des points critiques à l'infini.

Le premier succès notable de $\mathrm{A}$. BAHRI concerne la résolution de l'équation aux dérivées partielles non linéaire :

$$
-\Delta u=u^{\frac{n+2}{n-2}} \operatorname{sur} \Omega, u>0 \text { sur } \Omega, u=0 \text { sur } \partial \Omega \text { ò̀ } \Omega \text { est un domaine de } \mathbb{R}^{n} .
$$

Il montre (en collaboration avec J.M. Coron) que ce problème possède une solution si la topologie de $\Omega$ n'est pas triviale. La difficulté essentielle (qui avait arrêté de nombreux spécialistes) provient du manque de compacité dans la formulation variationnelle : la célèbre condition de Palais-Smale tombe en défaut et donc aucune méthode classique ne s'applique. L'idée nouvelle introduite pour contourner cette difficulté est éblouissante.

En gros, le problème est "compactifié" par une analyse très fine des suites de PalaisSmale qui "s'échappent à l'infini" - une méthode introduite pour la première fois par A. BAHRI dans son travail sur les formes de contact.

Le deuxième succès important de A. BAHRI concerne le problème des trois corps, classique en Mécanique Céleste. Très récemment, A. BAHRI et P. RABINOWITZ ont prouvé 
qu'il existe une infinité de solutions périodiques (généralisées) du problème des trois corps. Ces solutions sont associées au principe variationnel $\delta E=0$ où

$$
E=\sum_{i=1}^{3} \int_{0}^{1}\left|\dot{q}_{i}\right|^{2} \mathrm{~d} t+\sum_{i \neq j} \int_{0}^{1} \frac{\alpha_{i j}}{\left|q_{i}-q_{j}\right|} \mathrm{d} t \quad\left(\alpha_{i j}>0\right) .
$$

Il s'agit d'une étape très importante vers la solution d'un problème posé par H. Poincaré (pour arriver à une solution complète il reste encore à exclure les orbites avec collisions). La démonstration est un véritable tour de force combinant brillamment Topologie et Analyse. Ici encore l'idée maîtresse consiste à étudier les "points critiques à l'infini" pour contourner la condition de Palais-Smale qui tombe en défaut.

Ces exemples montrent que la méthode des "points critiques à l'infini" constitue un progrès fondamental en Calcul des Variations car de nombreux autres problèmes issus de la Géométrie Différentielle et de la Physique présentent des difficultés comparables. La théorie des "points critiques à l'infini" pourrait donc devenir un outil classique dans les années à venir.

Auteur de deux livres, A. BAHri a publié une dizaine de notes aux Comptes Rendus de l'Académie des Sciences et une dizaine d'articles dans les meilleures revues internationales. Il a été invité à faire des conférences dans les centres mathématiques les plus prestigieux (Princeton, Mittag-Leffler, Max Plank à Bonn, ETH à Zurich, MSRI à Berkeley, Courant Institute à New-York, École Normale de Pise, IMPA à Rio, Academia Sinica à Pékin, etc...). En plus de sa passion pour les mathématiques, A BAHRI s'intéresse beaucoup à la peinture et à la littérature. 